\title{
Jahn-Teller Effect of Cu-Ferrite Films by Solid Reaction
}

\author{
T. Tanaka, M. Chiba*, H. Okimura** and Y. Koizumi
}

Department of Applied Physics, Tokai University, 1117 Kitakaname, Hiratsuka, Kanagawa, 259-12 Japan

* Department of Material Science and Technology, Tokai University, 317 Nishino, Numazu, Shizuoka, 410-03 Japan

** Department of Metallurgical Engineering, Tokai University, 1117 Kitakaname, Hiratsuka, Kanagawa, 259-12 Japan

\begin{abstract}
Copper iron oxide ( $\mathrm{CuFe} \mathrm{O}_{4}: \mathrm{Cu}$-ferrite) thin film was prepared on a glass substrate by vacuum evaporation and solid reaction, and annealed at $400^{\circ} \mathrm{C}$ in the air. The obtained film was identified by $\mathrm{X}$-ray diffraction analysis as Cu-ferrite film having a tetragonal lattice. The axial ratio (c/a) of the film was 1.507 and was larger than that of ASTM data because the lattice distortion of the films seems to be large by difference of a preparation method. The temperature dependence of the magnetization and conductivities of the film showed remarkable changes at about $360^{\circ} \mathrm{C}$, which were considered to be caused by phase transition due to the Jahn-Teller effect.
\end{abstract}

\section{INTRODUCTION}

Copper iron oxide ( $\mathrm{CuFe}_{2} \mathrm{O}_{4}: \mathrm{Cu}$-ferrite) have a distinctive feature of the phase transition from a tetragonal to a cubic lattice at $360^{\circ} \mathrm{C}$ due to the Jahn-Teller effect $[1,2]$. It is the purpose of this study to point out the influence of the phase transition due to the Jahn-Teller effect on the magnetic properties and electrical conductivities.

\section{EXPERIMENT}

$\mathrm{Cu}$-ferrite film was prepared through the solid reaction described the following. Iron film of $200 \mathrm{~nm}$ thickness was evaporated onto heat-resisting glass substrate at room temperature using a vacuum evaporation method with an L-type electron beam heating gun under a pressure of $2 * 10^{6}$ Torr. The deposition rate was $0.2 \mathrm{~nm} / \mathrm{s}$. Iron film was oxidized to form $\alpha-\mathrm{Fe}_{2} \mathrm{O}_{3}$ film in the air at $400^{\circ} \mathrm{C}$ for 3 hours. Then, iron film of $40 \mathrm{~nm}$ thickness was evaporated onto $\alpha-\mathrm{Fe}_{2} \mathrm{O}_{3}$ film at room temperature, which was reduced to form $\mathrm{Fe}_{3} \mathrm{O}_{4} \mathrm{film}$ in a vacuum at $400^{\circ} \mathrm{C}$ for 6 hours. Next, copper film of $120.1 \mathrm{~nm}$ thickness was evaporated onto $\mathrm{Fe}_{3} \mathrm{O}_{4}$ film at room temperature, which was annealed in a vacuum at $400^{\circ} \mathrm{C}$ for 8 hours. Finally, $\mathrm{Fe}_{3} \mathrm{O}_{4}$ film included metallic copper was oxidized to form Cu-ferrite film in the air at $400^{\circ} \mathrm{C}$ for an hour. The thickness of Cu-ferrite film was about $850 \mathrm{~nm}$.

The thickness was measured after an evaporation by talystep. The crystal structure was identified by X-ray diffraction (XRD) analysis. The surface condition of the film was observed by scanning electron microscopy (SEM). The magnetic properties and electrical conductivities were measured by vibrating sample magnetometer (VSM) and two-point probe method, respectively.

\section{RESULTS AND DISCUSSION}

\subsection{Crystal structure and surface condition [3]}

By the result of the XRD analysis, Cu-ferrite film have a tetragonal lattice and the diffraction spectrum from (200), (211), (220) and $(400)$ faces are appeared. We compare the lattice constants between the value that calculated from diffraction spectrum and the data listed on ASTM files of Cu-ferrite. The values of the axial ratio (c/a) of the film and of ASTM data are 1.507 and 1.477, respectively. Consequently, the lattice distortion of the film seems to be large by difference of a preparation method.

For the purpose of investigating the surface texture of the film, it is observed by SEM. The grain structure is formed in the film, and the grain size is about $100 \mathrm{~mm}$.

\subsection{Magnetic properties}

The M-H curve of the film is measured by VSM, where the magnetic field is applied in-plane to the film, is shown in Fig. 1. The saturation magnetization and coercive force of the film are $2.4 \mathrm{kG}$ and $290 \mathrm{Oe}$. Because the diffraction spectra from (200) and (400) faces are appeared and the induction of the uniaxial magnetic anisotropy is expected, the magnetic field is applied perpen- 
dicular to the film. But the M-H curve is not almost observed. It is thought that the anisotropy to the perpendicular direction of the film is small because they are not c-axial dominant orientational film.

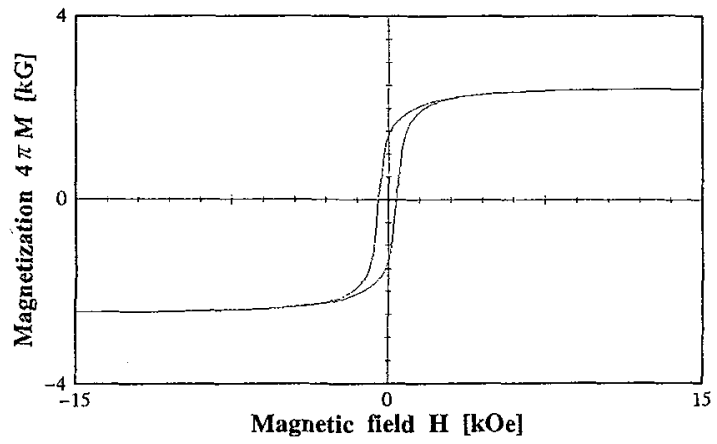

Figure 1: M-H curve of a Cu-ferrite film.

\subsection{Temperature dependence of the magnetization and conductivities}

For the purpose of investigating the influence of the phase transition due to the Jahn-Teller effect on the magnetic properties and electrical conductivities, the temperature dependence of the magnetization and conductivities of the film are measured and shown in Fig. 2 (a) and (b). The remarkable changes are observed at about $360^{\circ} \mathrm{C}$, which are considered to be caused by phase transition due to the Jahn-Teller effect. The Curic temperature $\mathrm{T}_{c}$ is about $460^{\circ} \mathrm{C}$. It is thought about the magnetization that the magnetic moment is influenced by gap of the band structure caused by phase transition. On the other hand, it is thought that the conductivities are changed by phase transition even though a similar material system because it is ruled by band structure decided by crystal structure.

The conduction type of a carrier is judged by thermoelectric power method. As a result, the film is the n-type semiconductor in the area of a tetragonal lattice. The conductivities are related to $\ln \left(s_{1} / s_{2}\right)=-\left(E_{g} / 2 k\right)\left(1 / T_{1}-1 / T_{2}\right)$. Here, $E_{g}$ is the energy gap, $k$ is the Boltzmann's constant and $\left(T_{1}, s_{1}\right),\left(T_{2}, s_{2}\right)$ are two voluntary points of the characteristic curve of the conductivities. If this relation is applied to a result of Fig. $2(\mathrm{~b}), E_{\mathrm{g}}$ is about $3.3 \mathrm{eV}$ in the area of a tetragonal lattice.
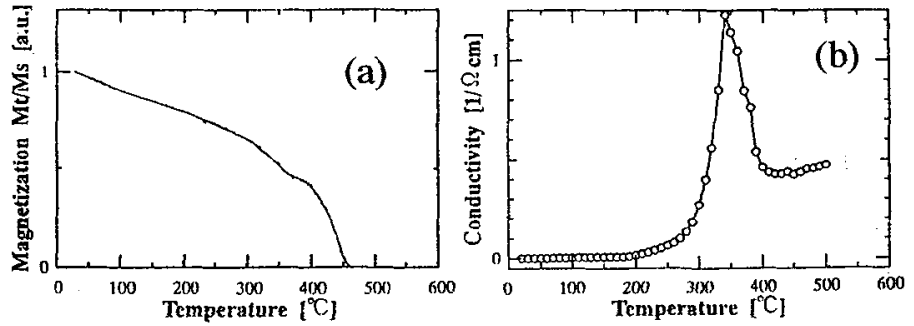

Figure 2: Temperature dependence of the magnetization and conductivity for the Cu-ferrite film.

\section{CONCLUSIONS}

Here we may draw the following conclusions: (1)The temperature dependence of the magnetization and conductivities show remarkable changes at $360^{\circ} \mathrm{C}$, which are considered to be caused by phase transition due to the Jahn-Teller effect. (2)The axial ratio (c/a) of the film is 1.507 and is larger than that of the data on ASTM files because the lattice distortion due to the Jahn-Teller effect is large. (3)The conductivities of the film show the characteristic of the n-type semiconductor in the area of a tetragonal lattice.

\section{References}

[1] Jahn H. A. and Teller E., Proc, Roy. Soc, A161, (1937)220-235.

[2] Finch G. I., Sinha A. P. B. and Sinha K. P., Proc. Roy. Soc., A242, (1957)28-35.

[3] Tanaka T., Fukae S., Chiba M., Okimura H. and Koizumi Y., J. Mag. Soc. Jpn., 20, (1996)265-268. 